\title{
Liga hipoeutética Al-11\%Si: coeficiente de transferência de calor interfacial, variáveis térmicas, parâmetros microestruturais e microdureza
}

\author{
Hypoeutectic Al-11wt.\%Si alloy: interfacial \\ heat transfer coefficient, thermal variables, \\ microstructural parameters and microhardness
}

\author{
José Augusto França Rodrigues ${ }^{1}$, Gueber Elias Mendes Santos Júnior ${ }^{1}$, \\ André dos Santos Barros ${ }^{1}$, Otávio Fernandes Lima da Rocha², \\ Antonio Luciano Seabra Moreira ${ }^{1}$
}

\footnotetext{
${ }^{1}$ Faculdade de Engenharia Mecânica, FEM/UFPA, Av. Augusto Corrêa 1, CEP: 66075-110, Belém, Pará, Brasil.

${ }^{2}$ Faculdade de Engenharia de Materiais, IFPA, Av. Almirante Barroso 1155, CEP: 66093-020, Belém, Pará, Brasil. e-mail: augustorodrigues95@hotmail.com,gueberjunior379@gmail.com, andre.barros13@hotmail.com, alsm@ufpa.br, otvrocha@gmail.com.
}

\begin{abstract}
RESUMO
Este artigo apresenta um estudo teórico-experimental com o objetivo de determinar o coeficiente de transferência de calor interfacial, as variáveis térmicas de solidificação, os espaçamentos dendríticos primários e secundários bem como a microdureza Vickers da liga Al-11\%Si solidificada direcionalmente, sob condições transientes de fluxo de calor, em um dispositivo de configuração horizontal refrigerado a água. Para o monitoramento dos perfis de temperatura utilizou-se um conjunto de termopares tipo K localizados em diferentes posições a partir da interface metal-molde, considerando a linha central correspondente ao eixo longitudinal do lingote. Uma técnica numérica, que compara perfis térmicos teóricos e experimentais, foi utilizada no cálculo do coeficiente de transferência de calor na interface metal/molde durante a solidificação cuja condição transiente é representada por uma equação analítica na forma de potência. Foram aplicadas técnicas experimentais para o cálculo das variáveis térmicas de solidificação, ou seja, deslocamento da frente de solidificação, velocidade da isoterma liquidus e taxa de resfriamento. Os resultados experimentais e simulados apresentaram uma concordância muito boa e possibilitaram estabelecer correlações analíticas entre tais variáveis térmicas e os espaçamentos dendríticos primários e secundários obtidos, por meio de equações na forma de potência representadas por expoentes característicos. Finalmente, são também apresentadas expressões experimentais capazes de correlacionar os valores dos espaçamentos dendríticos estudados com o comportamento da microdureza Vickers da referida liga.
\end{abstract}

Palavras-chave: Solidificação horizontal. Coeficiente de transferência de calor. Parâmetros térmicos. Microestrutura. Microdureza.

\begin{abstract}
This paper presents a theoretical-experimental study aiming to determine the interfacial heat transfer coefficient and solidification thermal parameters from experimentally obtained data correlating these with the primary and secondary dendritic spacings as well as microhardness during the horizontal solidification of the Al-11wt.\%Si alloy under transient heat flow conditions. For the experimental procedure, a water-cooled solidification device was used which allows the horizontal directional solidification and the monitoring of the temperature profiles in different positions from the metal-mold interface considering the center line corresponding to the longitudinal axis of the ingot. A numerical technique which compares theoretical and experimental thermal profiles has permitted the evaluation of the variation of metal-mold heat transfer coefficient along the solidification. The interfacial heat transfer coefficient of studied alloy is represented by a equation
\end{abstract}


which show the time dependence during the process. The experimental and calculated values have shown a very good agreement. Experimental techniques were applied to calculate the following solidification thermal variables: solidification front displacement, growth rate and cooling rate. The experimental results allowed establishing a quantitative correlation between such thermal variables and the studied structural parameters by means of power type equations represented by characteristic exponents. Finally, correlation between the values of both primary and secondary dendritic spacings found with Vickers microhardness behavior for the studied alloy is also presented.

Keywords: Horizontal solidification. Heat transfer coefficient. Thermal parameters. Microstructure. Microhardness.

\section{INTRODUÇÃO}

As ligas de alumínio possuem fundamental importância industrial, uma vez que das ligas oriundas de fundição as mesmas são as mais empregadas dentre todas as ligas não-ferrosas. De acordo com PERES et al. [1], as ligas Al-Si constituem a parcela mais significativa de todas as peças e componentes fundidos fabricados, sendo largamente utilizadas principalmente na indústria automotiva na fabricação, por exemplo, de cabeçotes, pistões e até blocos de cilindros sendo ainda esperado um significativo crescimento neste setor industrial nos próximos anos. Isto se deve principalmente ao excelente efeito que a adição de silício tem na fundibilidade, fluidez e redução de trincas no produto fundido, além de proporcionar melhoria das propriedades mecânicas e resistência à corrosão $[1,2]$. Em geral, as ligas desse sistema possuem uma faixa larga de acréscimo de silício, sendo de 5 a $7 \%$ para processos com baixas taxas de resfriamento (areia, gesso, por exemplo), de 7 a $9 \%$ para moldes permanentes e de 8 a $12 \%$ em peso para fundição sob pressão [3]. De modo particular, nos estudos recentemente desenvolvidos por KAKITANI et al. [4, 5], são apresentadas equações matemáticas que relacionam variáveis térmicas de solidificação com parâmetros microestruturais da liga Al-11\%Si durante a solidificação direcional vertical ascendente, incluindo ainda a adição de Ni como terceiro elemento. Entretanto, a caracterização da liga Al-11\%Si processada em sistemas de solidificação nos quais fluxos convectivos térmicos e solutais tendem a ser mais intensos ainda permanece como um campo aberto para pesquisa.

Por outro lado, considerando a correlação entre processo, estrutura, propriedades e desempenho de um material, o entendimento da relação entre parâmetros de solidificação e a estrutura resultante é fundamental para o desenvolvimento de novos processos que possibilitem a melhoria de propriedades requeridas, precisão dimensional e controle de qualidade de produtos fundidos [6-8]. Nesse sentido, é muito importante para o setor industrial o conhecimento dos mecanismos que controlam o processo de solidificação, objetivando a eficiência e a performance operacional dos mesmos. Desta forma, dentre as diversas condições existentes em tal processo, a transferência de calor e massa envolvidas na transformação de fase líquido/sólido possui grande destaque, tendo o coeficiente de transferência de calor interfacial $\left(\mathrm{h}_{\mathrm{i}}\right)$, principalmente nos instantes iniciais, como uma das variáveis térmicas que controlam de forma mais expressiva os parâmetros térmicos em que o fenômeno ocorre, uma vez que o contato do líquido com as paredes internas do molde ocasiona o surgimento de uma resistência de contato, de natureza térmica, conhecida como resistência newtoniana [9, 10]. No início do processo de solidificação, uma camada delgada de metal solidifica junto às paredes do molde com elevado grau de contato entre as duas superfícies, sendo que este contato diminui significativamente à medida que o processo avança em função, por exemplo, da contração do metal sólido e expansão do molde, de tal maneira a formar um espaço físico que promove a separação entre o metal e o molde. Logo, diversos estudos avaliam os mecanismos pelos quais o calor é transferido através da interface metal/molde durante a solidificação sob as mais variadas condições operacionais [11-13].

Este coeficiente $\left(\mathrm{h}_{\mathrm{i}}\right)$ juntamente com outros parâmetros operacionais de solidificação (composição da liga, superaquecimento, material do molde, presença de convecção termossolutal no líquido etc.) variam de acordo com o processo de fabricação adotado. Desta forma, tais parâmetros influenciam nas condições térmicas em que o fenômeno ocorre, como nas velocidades de deslocamento da isoterma liquidus $\left(\mathrm{V}_{\mathrm{L}}\right)$, nos gradientes de temperatura $\left(\mathrm{G}_{\mathrm{L}}\right)$ e nas taxas de resfriamento $\left(\mathrm{T}_{\mathrm{R}}\right)$. Tais condições transientes, bem como as diferentes composições das ligas, conduzem a diversas possibilidades de obtenção de estruturas finais para um mesmo produto fundido e, consequentemente, para o seu desempenho mecânico. Assim, um melhor entendimento da influência dos parâmetros térmicos sobre a formação dos aspectos estruturais é imprescindível para o planejamento dos processos de fundição e lingotamento com o intuito de serem obtidas propriedades mecânicas, resistência à corrosão e, até mesmo, resistência ao desgaste adequadas às peças obtidas [15].

As propriedades decorrentes do processo de fundição são fortemente influenciadas pela microestrutura. De acordo com ROOY [3], o grau de refinamento dos espaçamentos dendríticos é, muitas vezes, mais relevante que o próprio tamanho de grão macroestrutural para a melhoria das propriedades mecânicas de alguns 
sistemas metálicos. O crescimento dendrítico talvez seja o fenômeno mais frequentemente observado durante a solidificação cuja morfologia é caracterizada por espaçamentos de braços dendríticos primários $\left(\lambda_{1}\right)$, secundários $\left(\lambda_{2}\right)$ e terciários $\left(\lambda_{3}\right)$. Como o fluxo do líquido nos canais interdendríticos depende desses espaçamentos dendríticos, é muito importante conhecer a variação desses parâmetros durante o processo de solidificação para analisar a microssegregação que influencia, por exemplo, a cinética de homogeneização, limite de resistência à tração, ductilidade, tenacidade, limite de elasticidade, limite de resistência à fadiga, microdureza etc. Sabe-se que o grau de refino da estrutura dendrítica pode ser bem mais importante que o tamanho do grão para a melhoria das propriedades mecânicas, visto que a redução desse parâmetro estrutural permite uma distribuição mais uniforme de partículas de segundas fases, porosidade e inclusões [15-19]. Por isso, essas estruturas são consideradas como um importante objeto de investigação com relação à solidificação de ligas metálicas, uma vez que a referida estrutura é determinada pelas condições com que o calor é extraído durante a transformação líquido/sólido.

Os espaçamentos dendríticos são parte integrante da microestrutura exercendo, portanto, influência direta sobre as propriedades dos materiais fundidos. No caso da microdureza, por exemplo, estudos desenvolvidos por FAN et. al. [20] relatam esta propriedade como sendo uma maneira relativamente simples de realizar a complexa tarefa de prever algumas importantes propriedades mecânicas das ligas. Assim, para algumas ligas Ti-Al, a microdureza Vickers (HV) já vem sendo utilizada há algum tempo no controle de qualidade durante a produção de turbocompressores, válvulas automotivas e pás de compressores. Em estudos publicados anteriormente, KAYA et. al. [21] correlacionam os parâmetros microestruturais $\left(\lambda_{1}\right.$ e $\left.\lambda_{2}\right)$ de ligas à base de alumínio com valores de microdureza, medidos nas seções transversal e longitudinal do produto fundido sob condições de solidificação em regime estacionário. No caso do sistema Al-Si, o grau de refino dos espaçamentos dendríticos é determinante nos valores de microdureza uma vez que estruturas mais refinadas distribuem mais homogeneamente as partículas de silício as quais possuem maior resistência à deformação (indentação). O grau de refino microestrutural no sistema Al-Si também dificulta a movimentação de discordâncias aumentando, portanto, a dureza.

Neste contexto, com o intuito de se avaliar o desenvolvimento do metal sólido ao longo do processo, a técnica de solidificação direcional tem sido bastante utilizada em experimentos. Estes estudos referentes aos fenômenos envolvidos durante a transformação de fases objetiva a caracterização, principalmente, de aspectos relacionados às microestruturas obtidas. Tal técnica é amplamente empregada também por investigar os eventos físicos envolvidos somente em uma direção (x, y e z), estabelecendo princípios e equações que regem cada caso. Desta maneira, dependendo da direção assumida (vertical ou horizontal), o vetor gravidade influenciará de diferentes formas a direção de crescimento do sólido formado pois os efeitos impostos serão bastante diferentes para uma liga solidificada em função das diferentes condições operacionais assumidas. No caso do sistema vertical ascendente, a mudança de fase líquido/sólido acontece em sentido contrário ao da ação da gravidade com o peso próprio do lingote, portanto, favorecendo o contato térmico com a parede do molde. Durante o processo, pode ser observada a formação de um líquido interdendrítico podendo ser mais ou menos denso que o líquido de origem devido à rejeição de soluto. Tal líquido formado está localizado junto à região de transformação de fases. Devido a isto, correntes convectivas por diferença de temperaturas ou por diferença de concentrações de soluto não ocorrem, facilitando a análise da transferência de calor uma vez que esta acontece essencialmente por condução térmica [21-26]. No sistema vertical descendente, a extração de calor ocorre no topo do lingote. Neste caso, a solidificação acontece no mesmo sentido ao da ação gravitacional com o peso próprio do lingote atuando de forma a deslocá-lo do contato com o molde, caracterizando uma situação de maior resistência térmica à passagem de calor do lingote em direção ao meio ambiente. Devido ao crescente perfil de temperaturas em direção à base do lingote, pode-se notar que ocorrerá, pelo menos, convecção por diferença de temperatura no líquido [27, 28]. Quando considera-se o fenômeno da solidificação em um sistema direcional horizontal, isto é, quando o fluxo de calor é extraído através de uma das paredes laterais do molde, a convecção termossolutal e o efeito da gravidade estão presentes. Portanto, uma interessante característica adicional do sistema horizontal é o gradiente de concentração de soluto bem como os efeitos de densidade na direção vertical, uma vez que o líquido enriquecido de soluto, dependendo de sua densidade, pode decantar ou não, ao passo que o solvente tende a emergir devido as forças de flutuabilidade. Além disso, devido os efeitos impostos pela convecção termossolutal, sempre vai ocorrer um gradiente de temperaturas na direção vertical [14, 29-33]. Na literatura, observa-se que a grande maioria dos estudos desenvolvidos dedicam-se a investigar a formação de estruturas dendríticas obtidas durante a solidificação unidirecional de materiais metálicos somente nos sistemas verticais considerando o regime estacionário de extração de calor. Assim, poucos são os trabalhos que tratam da solidificação direcional em sistemas com configuração horizontal em regime transiente, visto que esta é mais complexa sob o ponto de vista da determinação das variáveis térmicas de solidificação. Considerando a importância do assunto, este estudo objetiva 
desenvolver um estudo teórico-experimental capaz de analisar a interrelação entre o coeficiente de transferência de calor interfacial, os parâmetros térmicos velocidade de deslocamento da isoterma liquidus e taxa de resfriamento, o comportamento dos espaçamentos dendríticos primários e secundários e a microdureza Vickers resultantes da liga Al-11\%Si solidificada direcionalmente em um sistema com configuração horizontal.

\section{MATERIAIS E MÉTODOS}

O dispositivo de solidificação unidirecional horizontal refrigerado a água utilizado na realização deste trabalho, ilustrado na Figura 1, foi projetado de tal maneira que a extração de calor do metal líquido ocorresse somente por meio de um sistema refrigerado a água situado em uma das paredes laterais do molde, resultando desta forma na solidificação transiente horizontal. O dispositivo é constituído de resistências elétricas que permitem controlar sua potência com o objetivo de estabilizar os diferentes níveis de superaquecimento no metal líquido, assim como propiciar um isolamento térmico adequado, evitando perdas de calor pelas laterais e pela base do molde. O molde de aço inoxidável utilizado possui $160 \mathrm{~mm}$ de comprimento, $60 \mathrm{~mm}$ de largura, $60 \mathrm{~mm}$ de altura e suas paredes $3 \mathrm{~mm}$ de espessura. Suas superfícies laterais internas foram revestidas com camadas de alumina e a parte superior foi isolada com material refratário para evitar perdas de calor para o meio ambiente.

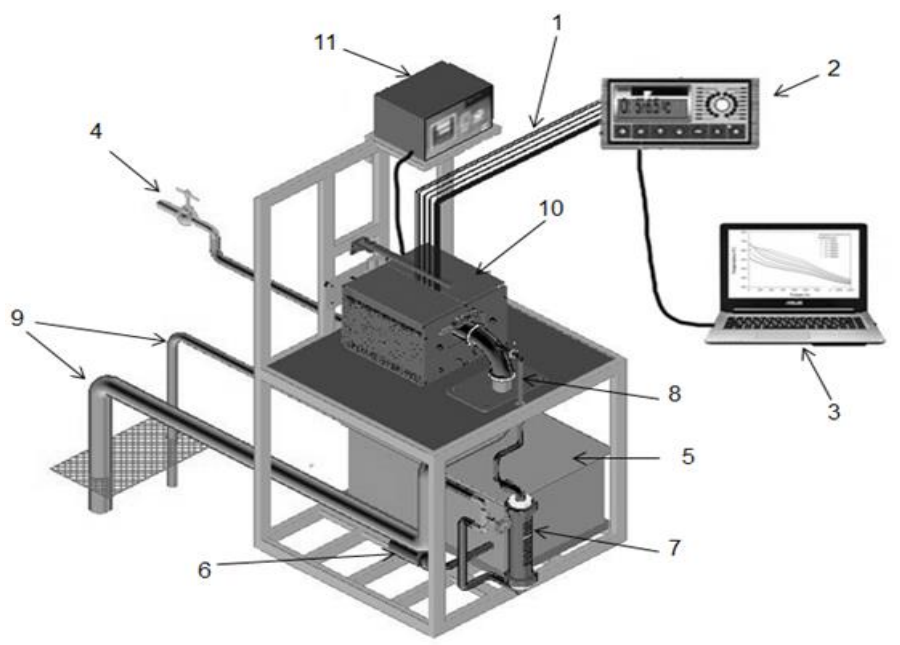

Figura 1: Dispositivo de solidificação horizontal utilizado, onde - 1) Termopares, 2) FieldLogger, 3) Computador, 4) Alimentação de água, 5) Armazenador de água, 6) Bomba d'água, 7) Rotâmetro, 8) Entrada de água, 9) Saída de água, 10) Dispositivo de solidificação horizontal, 11) Controlador de temperatura.

Inicialmente, a liga fundida foi vazada na lingoteira do dispositivo de solidificação. Nessa etapa, o aquecimento do dispositivo foi mantido até que fosse atingida a temperatura de trabalho correspondente a $10 \%$ acima da temperatura liquidus $\left(\mathrm{T}_{\mathrm{L}}\right)$ da liga. Alcançado o nível de superaquecimento estabelecido, as resistências elétricas do dispositivo foram então desligadas sendo imediatamente acionado o sistema de arrefecimento. Essa condição de resfriamento permaneceu até que o processo de solidificação fosse completado. O fluxo contínuo do fluido de arrefecimento à temperatura ambiente foi direcionado somente a uma das paredes laterais da lingoteira, induzindo uma extração de calor no sentido longitudinal da mesma, ou seja, apenas na direção horizontal.

O monitoramento do processo de solidificação e o correspondente registro térmico foram realizados com auxílio de seis termopares inseridos em posições predefinidas no interior da lingoteira e conectados ao sistema de aquisição de dados do dispositivo. A escolha dos termopares do tipo K ocorreu em função da larga faixa de utilização dos mesmos, o que permitiu sua utilização neste estudo. Através dos oito termopares posicionados a 5, 10, 15, 20, 30 e $50 \mathrm{~mm}$ a partir da superfície de extração de calor, todos conectados por meio de cabos coaxiais a um registrador de dados interligado a um computador, os valores das temperaturas foram medidos durante o processo de solidificação. Os registros dessas temperaturas foram realizados mediante o uso do software FieldLogger integrado ao dispositivo de solidificação, que digitaliza as medições dos termopares em tempo real pelo sistema de aquisição de dados, sendo obtidos os pares de decrescimento da temperatura com o passar do tempo $(\mathrm{T}, \mathrm{t})$ de cada termopar. Com o uso do software Origin Pro 8.0 plotaram-se os perfis térmicos de resfriamento das ligas, a partir dos quais foram levantadas as respectivas curvas dos parâ- 
metros térmicos de interesse.

Para a simulação do coeficiente de transferência de calor interfacial $\left(\mathrm{h}_{\mathrm{i}}\right)$ as propriedades termofísicas da liga investigada foram utilizadas, conforme a Tabela 1. Para tal simulação, utilizaram-se as propriedades mencionadas para fornecer um perfil térmico simulado pelo programa e compara-lo com aquele coletado durante o experimento [34].

Tabela 1: Propriedades termofísicas utilizadas na simulação do coeficiente interfacial $h_{\mathrm{i}}$.

\begin{tabular}{l|c|c|c|c|c}
\hline $\begin{array}{l}\text { PROPRIEDADE } \\
\text { TERMOFÍSICA }\end{array}$ & SímBOLO & UNIDADE & Al-11\%Si & AI PURO & $\begin{array}{c}\text { Al-12,6\%Si } \\
\text { (EUTÉTICO) }\end{array}$ \\
\hline \multirow{2}{*}{$\begin{array}{l}\text { Condutividade térmi- } \\
\text { ca }\end{array}$} & $\mathrm{k}_{\mathrm{S}}$ (sólido) & $\mathrm{W} / \mathrm{m} \mathrm{K}$ & 174,36 & 221,6 & 165,92 \\
\cline { 2 - 6 } & $\mathrm{k}_{\mathrm{L}}$ (líquido) & $\mathrm{W} / \mathrm{m} \mathrm{K}$ & 87,32 & 91,9 & 86,5 \\
\hline \multirow{2}{*}{ Calor específico } & $\mathrm{c}_{\mathrm{S}}$ (sólido) & $\mathrm{J} / \mathrm{kg} \mathrm{K}$ & 1191,38 & 1255 & 1180 \\
\cline { 2 - 6 } & $\mathrm{c}_{\mathrm{L}}$ (líquido) & $\mathrm{J} / \mathrm{kg} \mathrm{K}$ & 1196,21 & 1175 & 1200 \\
\hline \multirow{2}{*}{\begin{tabular}{c} 
Massa específica \\
\cline { 2 - 6 }
\end{tabular}} & $\rho_{\mathrm{S}}$ (sólido) & $\mathrm{kg} / \mathrm{m}^{3}$ & 2449,71 & 2559 & 2430,175 \\
\hline Calor latente de fusão & $\mathrm{L}$ & $\mathrm{kg} / \mathrm{m}^{3}$ & 2405,19 & 2389 & 2408,08 \\
\hline Temperatura liquidus & $\mathrm{T}_{\mathrm{L}}$ & $\mathrm{J} / \mathrm{kg}$ & 479833,16 & 395000 & 495000 \\
\hline Temperatura eutética & $\mathrm{T}_{\mathrm{E}}$ & ${ }^{\circ} \mathrm{C}$ & 590 & $\mathrm{Tf}=660$ & - \\
\hline $\begin{array}{l}\text { Coeficiente de parti- } \\
\text { ção de soluto }\end{array}$ & $\mathrm{k}_{0} \mathrm{C}$ & 577 & - & 577 \\
\hline $\begin{array}{l}\text { Inclinação da linha } \\
\text { liquidus }\end{array}$ & $\mathrm{m}_{\mathrm{L}}$ & - & 0,13 & - & - \\
\hline
\end{tabular}

Os valores obtidos por meio dos oito termopares foram utilizados para gerar uma equação da posição, a partir da interface metal/molde, em função do tempo, ou seja, $\mathrm{P}=\mathrm{f}(\mathrm{t})$, correspondente à passagem da isoterma liquidus em cada termopar. O procedimento baseou-se na interseção de uma reta correspondente à temperatura liquidus $\left(\mathrm{T}_{\mathrm{L}}\right)$ da liga investigada com os vários perfis térmicos gerados por cada termopar em sua respectiva posição. Assim, os valores levantados foram capazes de representar o tempo de passagem da isoterma liquidus em cada posição de termopar. Os pares ordenados posição $\mathrm{x}$ tempo $(\mathrm{P}, \mathrm{t})$ permitiram que fosse traçado um gráfico experimental da posição da isoterma liquidus com o tempo. Determinada a expressão algébrica da posição em função do tempo, foi calculada a velocidade experimental da isoterma liquidus $\left(V_{L}\right)$ em função de cada posição através da derivada dessa equação.

Os valores das taxas de resfriamento $\left(T_{R}\right)$, foram estimados por meio de derivadas de funções polinomiais de segunda ordem $T=f(t)$ plotadas sobre dados experimentais compreendidos em intervalos imediatamente anteriores e posteriores aos respectivos pontos de interseção da linha horizontal, indicativa da $T_{L}$, com os perfis de resfriamento gerados pelos termopares.

Após a realização dos diversos seccionamentos transversais e longitudinais necessários, diversas amostras da liga foram obtidas nas posições correspondentes a 2, 4, 6, 10, 15, 20, 30, 40, 50, 70, 90 e 110 $\mathrm{mm}$ em relação à interface metal/molde e embutidas a frio para posterior análise metalográfica. Em seguida, essas amostras foram lixadas sequencialmente por lixas de granulometrias 100, 220, 320, 400, 600 e 1200 mesh em lixadeiras rotativas. Com as amostras lixadas e limpas, as mesmas foram polidas com pasta de diamante e pano para polimento de 1 a $3 \mu \mathrm{m}$ em politriz rotativa, o que permitiu perfeitas condições às superfícies de serem atacadas quimicamente. A revelação das microestruturas ocorreu mediante imersão das amostras em uma solução aquosa ácida de $0,5 \%$ de HF durante 25 segundos [1].

Concluída a etapa metalográfica, as referidas amostras foram então analisadas microscopicamente com auxílio do sistema de processamento de imagens Olimpus, modelo UC30, acoplado ao software de captura de imagem Analise Sys 5.1. As imagens foram importadas para o software ImageJ para finalmente serem realizadas as medições dos espaçamentos dendríticos primários e secundários da liga. Nas medições dos espaçamentos primários foram consideradas seções transversais, portanto perpendiculares ao fluxo de calor, somente ao longo da região colunar. A medição dos espaçamentos secundários considerou as seções longitudinais, isto é, paralelas ao fluxo de calor. As técnicas adotadas para medição desses espaçamentos dendríticos $\left(\lambda_{1}\right.$ e $\left.\lambda_{2}\right)$ encontram-se na literatura $[33,35]$. Foram realizadas vinte medidas para cada posição considerada. 
Os ensaios de microdureza foram realizados utilizando-se uma carga de 50 gf durante um tempo de 10 segundos, conforme estabelece a Norma ASTM E384. Foram realizadas 20 medições em cada amostra analisada ao longo do comprimento do lingote, considerando o centro como referência e respeitando-se uma distância de 0,75 mm entre as indentações. As medições de microdureza foram obtidas em duas regiões da microestrutura de cada amostra, ou seja, no centro das dendritas, assim como em pontos da região interdendrítica. De posse dos resultados experimentais, foi possível levantar os perfis de microdureza pelo método Vickers para a liga $\mathrm{Al}-11 \% \mathrm{Si}$, utilizando-se o software Origin Pro 8.0, correlacionando-os as posições $(\mathrm{P}) \mathrm{com}$ as variáveis térmicas velocidade da isoterma liquidus $\left(\mathrm{V}_{\mathrm{L}}\right)$ e taxa de resfriamento $\left(\mathrm{T}_{\mathrm{R}}\right)$, bem como com os espaçamentos dendríticos primários e secundários apresentados por meio de uma variação entre os limites mínimo e máximo das 20 diferentes medições realizadas. Os testes de microdureza realizados neste trabalho foram obtidos a partir do dispositivo de microdureza modelo HMV-2, marca Shimadzu.

\section{RESULTADOS E DISCUSSÃO}

\subsection{Perfis Térmicos e Variáveis de Solidificação}

Os perfis térmicos experimentais e simulados referentes aos seis termopares inseridos no metal líquido durante a solidificação da liga investigada são apresentados na Figura 2, assim como a equação de $\mathrm{h}_{\mathrm{i}}$ em função do tempo. Pode-se observar que nos estágios inicias durante o experimento, os valores de $h_{i}$ são bem mais elevados devido a boa conformidade superficial entre a camada de metal solidificada inicialmente e o molde. À medida que a solidificação progride, o molde se expande devido à absorção de calor e, simultaneamente, o metal sólido contrai devido ao próprio fenômeno da mudança de fases. Em consequência, na interface de contato do metal solidificado com a chapa molde através da qual o calor é extraído, a pressão imposta pelo metal líquido torna-se insuficiente para proporcionar uma boa condição de contato entre tais superfícies, proporcionando desta forma o surgimento de vazios na interface metal-molde preenchidos com ar diminuindo a transferência de calor rapidamente, tornando os valores de $h_{i}$ praticamente constantes. Entretanto, o $h_{i}$ pode ser influenciado por uma série de outros fatores, como o grau de fluidez da liga, o intervalo de solidificação, a direção em que ocorre a solidificação, as propriedades termofísicas etc. Assim, uma justificativa detalhada para o comportamento deste parâmetro deve ser bastante cuidadosa [14]. Logo, os coeficientes experimentais de transferência de calor são válidos para condições particulares nas quais são mensurados, de tal maneira que servem somente como referência no modelamento da solidificação de ligas.

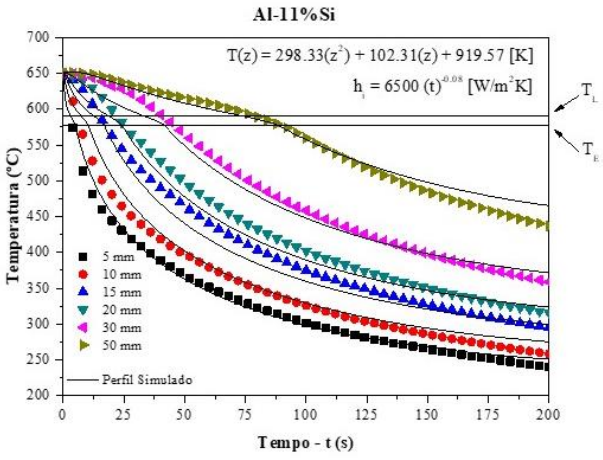

(a)

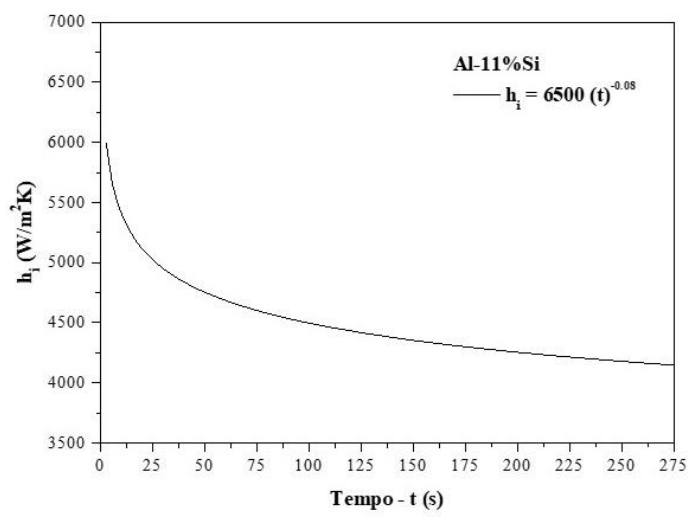

(b)

Figura 2: (a) Confronto entre os perfis térmicos experimentais e simulados e (b) Perfil do coeficiente de transferência de calor interfacial da liga $\mathrm{Al}-11 \% \mathrm{Si}$ solidificada nas condições assumidas.

Atualmente, sabe-se que os parâmetros microestruturais (espaçamentos celulares e dendríticos) dependem de parâmetros térmicos de solidificação, como $V_{L}$ e $T_{R}$, que variam com o tempo e a posição durante a transformação de fases. Com a intenção de se examinar a correlação existente entre tais parâmetros térmicos e o crescimento dendrítico durante a solidificação direcional horizontal sob condições transientes de fluxo de calor da liga $\mathrm{Al}-11 \% \mathrm{Si}$, os resultados obtidos a partir dos perfis térmicos experimentais foram utilizados na determinação desses parâmetros. Desta forma, os dados de cada curva do perfil térmico correspondente a cada posição definida a partir da interface metal/molde em função do tempo foram utilizados para deter- 
minar o avanço da frente de solidificação em cada termopar. Uma técnica de ajuste de curva nesses pares ordenados experimentais posição-tempo gerou uma função do tipo potência da posição experimental em função do tempo necessário até que a frente de solidificação atingisse determinado termopar, conforme apresentado na Figura 3. A partir da equação que correlaciona $h_{i}$ em função do tempo apresentada na Figura 2, obteve-se a curva posição versus tempo teórica a qual é comparada com os pontos experimentais como também é apresentada na Figura 3. Percebe-se que o ajuste nas posições iniciais do lingote é bem mais preciso que nas posições finais, uma vez que o coeficiente de transferência de calor interfacial possui grande influência no início da solidificação como descrito anteriormente e que a medida que o sólido cresce, as outras condições existentes na solidificação direcional horizontal passam a ter predomínio sobre tal crescimento.

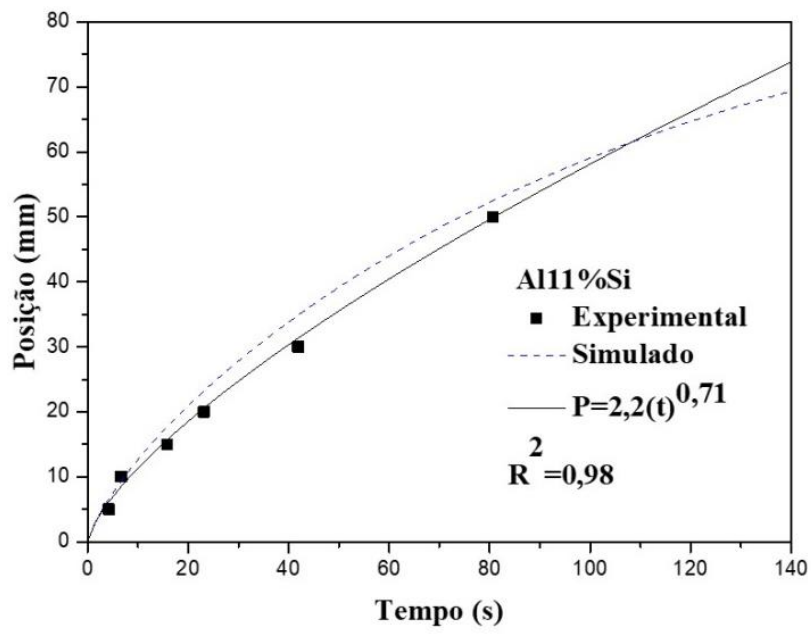

Figura 3: Deslocamento da isoterma liquidus em função do tempo.

A derivada da função posição em relação ao tempo produziu a equação analítica de velocidade de deslocamento da isoterma liquidus $\left(\mathrm{V}_{\mathrm{L}}\right)$ em relação à posição, conforme apresentado na Figura 4a. Como explicado anteriormente, a equação que descreve o comportamento da taxa de resfriamento ao longo da solidificação $\left(T_{R}\right)$, apresentada na Figura 4b, foi obtida através do método das derivadas. Em ambos os gráficos verifica-se também uma boa concordância entre as curvas experimentais e teóricas. Ao observar-se os dois gráficos, percebe-se que à medida que a camada de metal sólido aumenta, tanto $\mathrm{V}_{\mathrm{L}}$ como $\mathrm{T}_{\mathrm{R}}$ diminuem bruscamente até se estabilizarem um pouco antes da metade do comprimento do lingote devido à resistência térmica promovida por tal camada à extração de calor $[13,36]$. Percebe-se, também, que a concordância entre os pontos experimentais e os fornecidos pela simulação do método numérico foi bastante satisfatória.

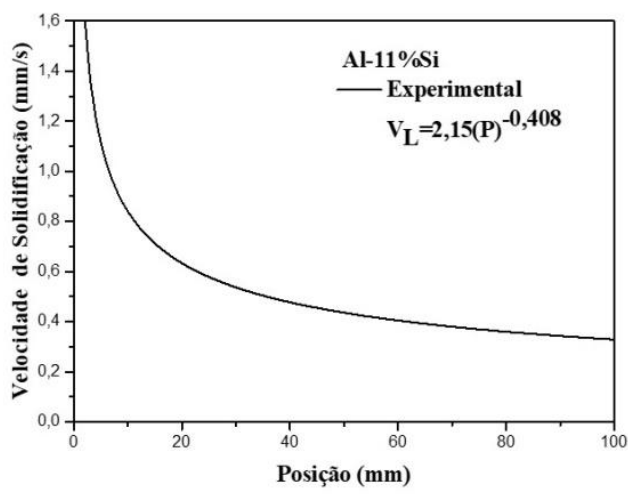

(a)

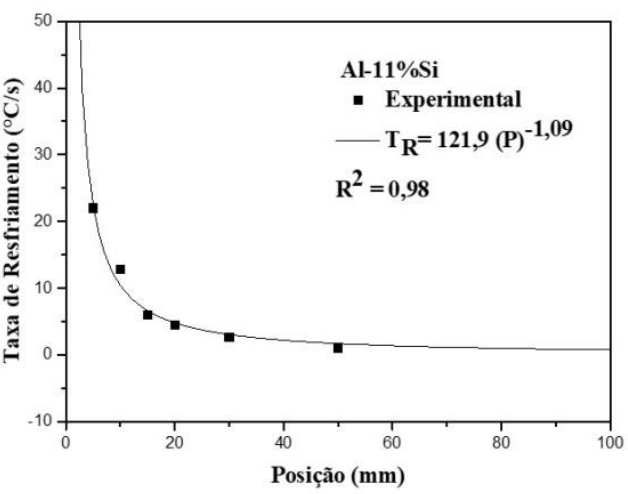

(b)

Figura 4: (a) Velocidade da isoterma liquidus e (b) taxa de resfriamento, ambas em função da posição.

\subsection{Avaliação Estrutural}

A macroestrutura resultante da solidificação, sob as condições operacionais assumidas nesse estudo, é apresentada na Figura 5, na qual se observa o crescimento de grãos colunares paralelamente à direção do flu- 
xo de extração de calor demonstrando assim, que a extração de calor foi efetivamente retirada na direção horizontal. A transição estrutural na liga $\mathrm{Al}-11 \% \mathrm{Si}$ começa em torno de $50 \mathrm{~mm}$ na parte inferior e encerra-se, aproximadamente, em $70 \mathrm{~mm}$ na parte superior do lingote, sendo todas essas dimensões em relação a interface metal/molde.

No que se refere ao formato apresentado pela macroestrutura, pode-se notar a presença de uma zona de transição estrutural de grãos colunares para grãos equiaxiais (TCE) em vez de uma transição brusca como ocorreu em alguns casos para outros teores de ligas desse sistema e em diferentes configurações de extração de calor [1, 27, 30]. A ocorrência da TCE nesta forma pode estar relacionada à existência de um perfil de gradiente térmico na direção vertical (direção da gravidade), assim como a de diferença de concentração de soluto tanto na direção de crescimento do sólido (horizontal) como na direção gravitacional (vertical).

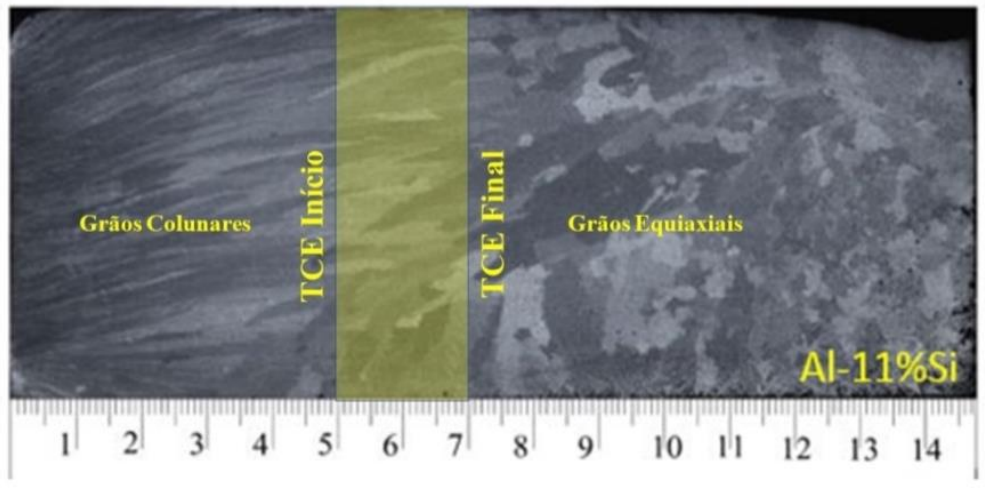

Figura 5: Macroestrutura de solidificação da liga estudada com a presença de TCE.

Em relação às microestruturas de solidificação pode-se notar, claramente, que as mesmas apresentam morfologia dendrítica, conforme apresentado na Figura 6. As seções transversais obtidas possibilitam a visualização de braços dendríticos primários, secundários formados na região de grãos colunares durante o processo de solidificação. Segundo pode ser observado, um aumento dos espaçamentos dendríticos tanto primário como secundário conforme a frente de solidificação se distancia da interface metal $/ \mathrm{molde}$. Tal fenômeno está diretamente associado à redução da velocidade de solidificação nas áreas mais distantes da interface metal/molde devido ao crescimento do próprio sólido que provoca uma resistência térmica.

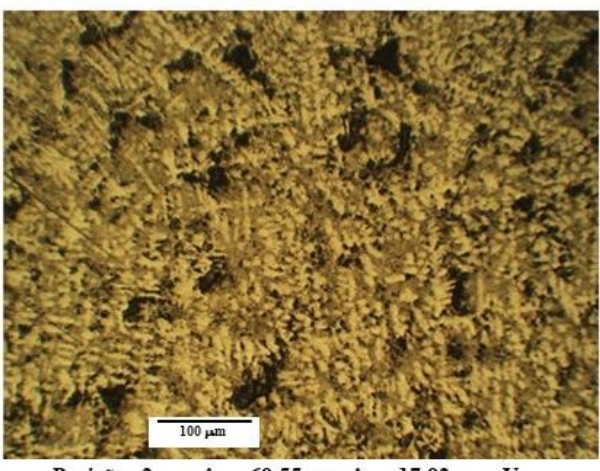

Posição: $2 \mathrm{~mm} ; \lambda_{1}=69,55 \mu \mathrm{m} ; \lambda_{2}=17,92 \mu \mathrm{m} ; \mathrm{V}_{\mathrm{L}}=$ $1,62 \mathrm{~mm} / \mathrm{s} ; \mathrm{T}_{\mathrm{R}}=56,86^{\circ} \mathrm{C} / \mathrm{s}$

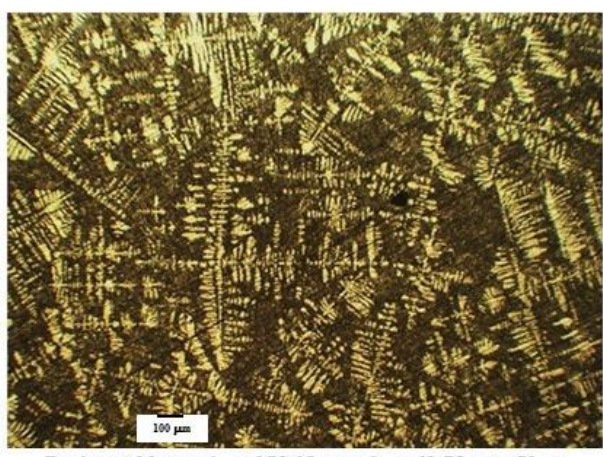

Posição: $30 \mathrm{~mm} ; \lambda_{1}=358,01 \mu \mathrm{m} ; \lambda_{2}=62,78 \mu \mathrm{m} ; \mathrm{V}_{\mathrm{L}}=$ $0,54 \mathrm{~mm} / \mathrm{s} ; \mathrm{T}_{\mathrm{R}}=2,89^{\circ} \mathrm{C} / \mathrm{s}$

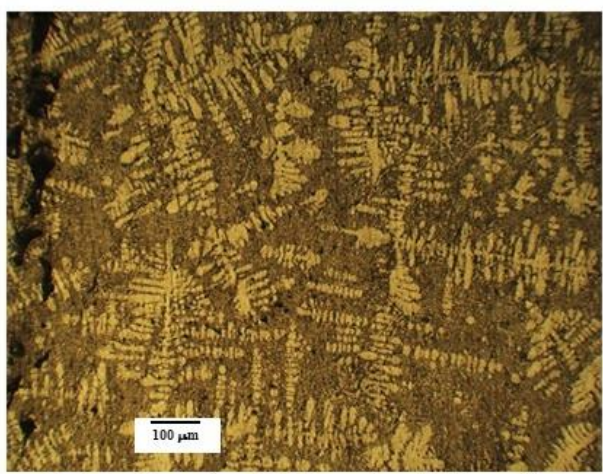

Posição: $15 \mathrm{~mm} ; \lambda_{1}=235,38 \mu \mathrm{m} ; \lambda_{2}=45,55 \mu \mathrm{m} ; \mathrm{V}_{\mathrm{L}}=$ $0,71 \mathrm{~mm} / \mathrm{s} ; \mathrm{T}_{\mathrm{R}}=6,20^{\circ} \mathrm{C} / \mathrm{s}$

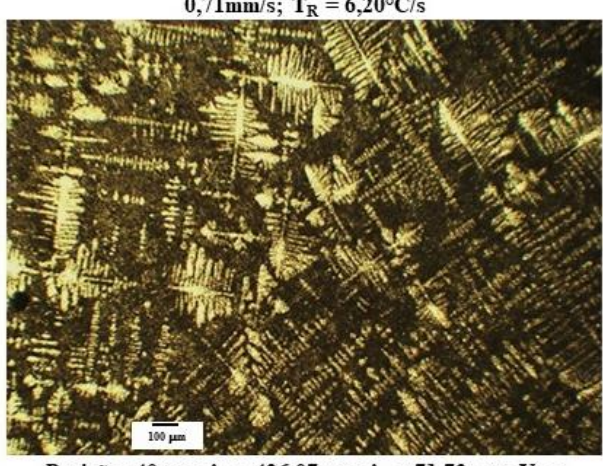

Posição: $40 \mathrm{~mm} ; \lambda_{1}=426,07 \mu \mathrm{m} ; \lambda_{2}=71,73 \mu \mathrm{m} ; \mathrm{V}_{\mathrm{L}}=$ $0,48 \mathrm{~mm} / \mathrm{s} ; \mathrm{T}_{\mathrm{R}}=2,11^{\circ} \mathrm{C} / \mathrm{s}$ 
Figura 6: Microestruturas dendríticas de solidificação da liga Al-11\%Si em diferentes posições em relação a interface metal-molde.

A Figura 7 apresenta os valores experimentais dos espaçamentos dendríticos $\left(\lambda_{1}\right.$ e $\left.\lambda_{2}\right)$ da liga investigada em função da velocidade de deslocamento da isoterma liquidus. Observa-se que ao passo que a velocidade $\left(\mathrm{V}_{\mathrm{L}}\right)$ aumenta, os espaçamentos diminuem. Além disto, pode-se verificar também que o expoente $-1,1$ para $\lambda_{1}$ e $\lambda_{2}$ foi determinado para a liga estudada, caracterizando desta maneira uma equação experimental para os espaçamentos dendríticos primários com $V_{L}$ na forma $\lambda_{1}, \lambda_{2}=$ Constante $\left(V_{L}\right)^{-1,1}$. No caso de $\lambda_{2}$, podese notar que após a TCE, os mesmos mudam de inclinação, de tal maneira que o expoente característico $\left(\lambda_{2}=\right.$ Constante $\left.\left(\mathrm{V}_{\mathrm{L}}\right)^{-2 / 3}\right)$ deste grau de espaçamentos não apresentou resultados satisfatórios. Já para $\lambda_{1}$, vale destacar que na literatura diversos trabalhos validam tal expressão (que relaciona $\lambda_{1}$ e $V_{L}$ ) para os mais variados sistemas de ligas. Para ligas do sistema Al-Cu, BARROS et. al. [14] e ROSA et. al. [28] determinaram a mesma relação experimental mencionada anteriormente com destaque para ROSA et. al. [28] que correlacionou também para ligas dos sistemas Al-Fe, Pb-Sn, Sn-Pb. Já para ligas Al-Si, PERES et al. [1] e CARVALHO et. al. [30] correlacionaram os referidos espaçamentos dendríticos com a velocidade de solidificação. Mais recentemente, BARBOSA et. al. [33] obteve o mesmo expoente para ligas do sistema ternário Al-Si$\mathrm{Mg}$, corroborando os resultados encontrados neste estudo para condições transientes de extração de calor.

Os resultados experimentais dos espaçamentos dendríticos primários das ligas estudadas também foram correlacionados com as taxas de resfriamento $\left(T_{R}\right)$, conforme mostrado na Figura 8. De forma idêntica ao que acontece com a relação velocidade de solidificação e espaçamentos dendríticos primário e secundário, os referidos espaçamentos também diminuem para valores mais elevados de $T_{R}$. Além de se poder observar também que a função é regida pela equação de crescimento dendrítico já consolidada e comprovada em diversos estudos existentes na literatura, $\lambda_{1}=$ Constante $\left(T_{R}\right)^{-0,55}$ para o primário e $\lambda_{2}=$ Constante $\left(T_{R}\right)^{-1 / 3}$ para o secundário.

É perceptível que os maiores valores de velocidades das isotermas liquidus e de taxas de resfriamento resultam em menores valores dos espaçamentos dendríticos, motivo pelo qual se observam um refinamento da estrutura dendrítica no início do processo de solidificação. Esta variação dos parâmetros estruturais (espaçamentos dendríticos) em função de parâmetros térmicos de solidificação $\left(V_{L}\right.$ e $\left.T_{R}\right)$ permite a previsão do comportamento microestrutural durante a solidificação horizontal da liga estudada, o que possibilita a programação da estrutura final do material a partir da definição dos valores impostos nas variáveis térmicas de solidificação [30].

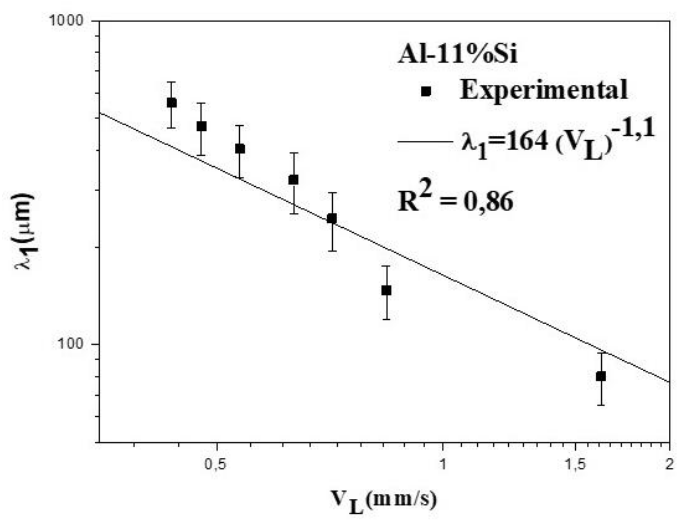

(a)

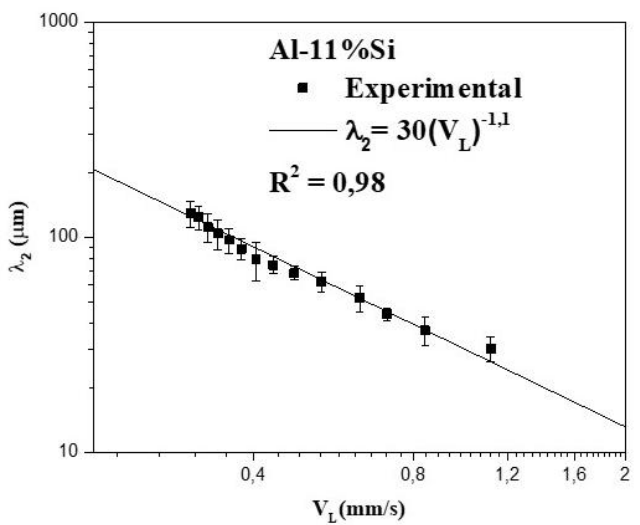

(b)

Figura 7: Espaçamentos dendríticos em função da velocidade - (a) primário e (b) secundário da liga estudada. 


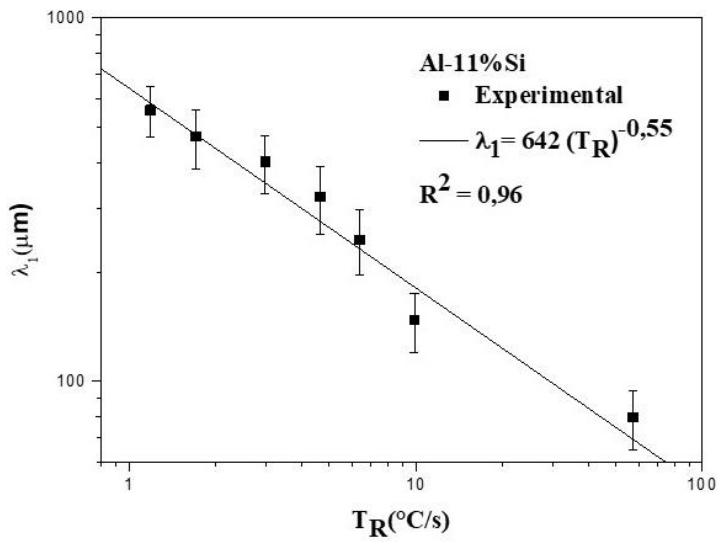

(a)

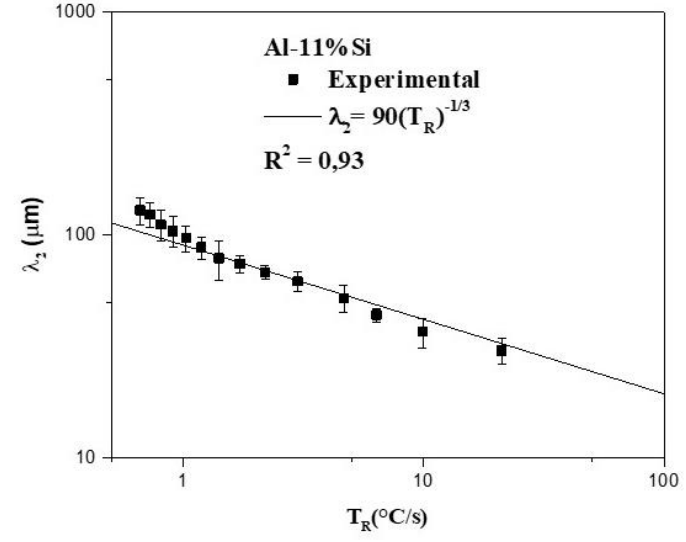

(b)

Figura 8: Espaçamentos dendríticos em função da taxa de resfriamento - (a) primário e (b) secundário da liga analisada.

Na Figura 9, a variação da microdureza Vickers (HV) é representada em função dos espaçamentos primários e secundários para a liga investigada, permitindo-se observar a redução dos valores de HV à medida que ocorre o distanciamento da interface metal/molde. Esta diminuição da microdureza está diretamente correlacionada com alterações microestruturais decorrentes da transformação líquido/sólido, principalmente com o engrossamento da estrutura dendrítica em função do crescimento do sólido, conforme indicado por alguns autores [14, 21, 37]. Tal comportamento é justificado pelo fato de que o refinamento dos espaçamentos dendríticos impõe maior dificuldade à movimentação de discordâncias durante a deformação (indentação), justificando a obtenção dos maiores valores de microdureza para posições próximas a interface de resfriamento $[14,33,38]$. De maneira geral, pode-se notar a tendência de elevação da microdureza com a diminuição dos valores tanto de $\lambda_{1}$ como de $\lambda_{2}$. Os valores de microdureza encontrados neste trabalho são relativamente próximos aqueles encontrados por KAKITANI et al. [4]. Todavia, enquanto KAKITANI et al. [4] adotaram um valor médio de microdureza $(48 \mathrm{HV})$ para a liga $\mathrm{Al}-11 \% \mathrm{Si}$, o presente trabalho propõe equações matemáticas para descrever a variação da microdureza HV em função de $\lambda_{1}$ e de $\lambda_{2}$. Observa-se que equações experimentais do tipo potência podem ser aplicadas para representar $\mathrm{HV}=\mathrm{f}\left(\lambda_{1}\right)$ bem como $\mathrm{HV}=$ $\mathrm{f}\left(\lambda_{2}\right)$ tendo em vista que para todos os casos foram encontrados $\mathrm{R}^{2}>0,7$.

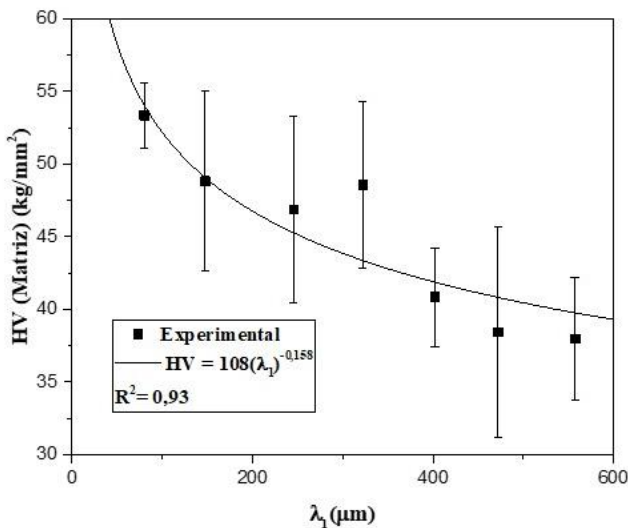

(a)

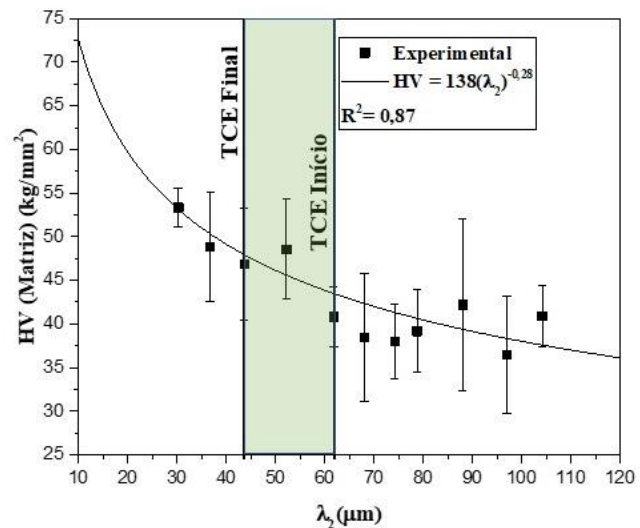

(b)

Figura 9: Microdureza Vickers em função dos - (a) espaçamentos dendríticos primários e (b) espaçamentos dendríticos secundários. 


\section{CONCLUSÕES}

A partir da análise dos resultados obtidos, é possível que sejam extraídas as seguintes conclusões:

a. A concordância entre os pontos experimentais fornecidos pelos dados térmicos durante a solidificação e as curvas obtidas pelo modelo teórico foi bem satisfatória, como observado nos ajustes dos perfis térmicos experimentais e teóricos. Pode-se notar também que no início do processo o $\mathrm{h}_{\mathrm{i}}$ possui uma influência muito mais predominante do que no término da solidificação.

b. Os parâmetros térmicos $V_{L}$ e $T_{R}$ foram determinados satisfatoriamente, sendo que os mesmos diminuem com o avanço da isoterma liquidus em função do aumento da resistência térmica promovida pela progressiva formação do metal sólido. Assim, quanto mais próximo da base refrigerada, maiores são os valores desses parâmetros.

c. A macroestrutura foi condizente com o esperado pela solidificação horizontal, apresentando grãos colunares bem definidos na direção de extração de calor pelo dispositivo de solidificação. Observa-se também que ocorre uma transição colunar-equiaxial (TCE) em uma zona do lingote a partir de $50 \mathrm{~mm}$ provocando fortes modificações nos espaçamentos secundários e, consequentemente, na microdureza da liga analisada.

d. A morfologia na liga apresentou-se como dendrítica, característico das altas taxas de extração de calor dos moldes refrigerados a água. A quantificação dos espaçamentos primários e secundários $\left(\lambda_{1}\right.$ e $\left.\lambda_{2}\right)$ da liga estudada permite concluir que uma vez que a ação do fluido de refrigeração impõe valores de $V_{L}$ e $T_{R}$ bastante elevados próximo à interface metal/molde, influenciam diretamente os espaçamentos dendríticos determinados experimentalmente, de maneira a proporcionar menores valores destes próximos à interface metal/molde.

e. Os expoentes $-1,1$ e $-0,55$ (para $\lambda_{1}$ ) $-1,1$ e $-1 / 3$ (para $\lambda_{2}$ ) caracterizam as leis experimentais de crescimento dos espaçamentos dendríticos primários e secundários em função da velocidade de solidificação e da taxa de resfriamento, respectivamente. Pode-se observar que a transição colunar-equiaxial influencia fortemente no comportamento dos espaçamentos dendríticos, de forma mais evidente nos espaçamentos secundários.

f. Equações experimentais do tipo potência foram capazes de correlacionar os resultados de microdureza com os referidos espaçamentos dendríticos primários e secundários evidenciando a relação de que à medida que os referidos espaçamentos diminuem, os valores de HV aumentam consideravelmente.

\section{BIBLIOGRAFIA}

[1] PERES, M.D., SIQUEIRA, C.A., GARCIA, A., "Macrostructural and microstructural development in AlSi alloys directionally solidified under unsteady-state conditions", Journal of Alloys and Compounds, v. 381, n. 1-2, pp. 168-181, Nov. 2004.

[2] DJURDJEVIČ, M.B., GRZINČIČ, M.A., "The effect of major alloying elements on the size of the secondary dendrite arm spacing in the as-cast Al-Si-Cu alloys" Archives of Foundry Engineering, v. 12, n. 1, pp. 19-24, Mar. 2012.

[3] ROOY, E.L., Metals Handbook, 9 ed., Ohio, Materials Park, 1988.

[4] KAKITANI, R., CRUZ, C., LIMA, T., et al. "Transient directional solidification of a eutectic Al-Si-Ni alloy: Macrostructure, microstructure, dendritic growth and hardness", Materialia, v. 7, pp. 100358, Sep. 2019.

[5] KAKITANI, R., GARCIA, A., CHEUNG, N., "Morphology of intermetallics tailoring tensile properties and quality index of a eutectic Al-Si-Ni alloy", Advanced Engineering Materials, v. 22, n. 12, pp. 2000503, Jul. 2020.

[6] FLEMINGS, M.C., Solidification processing. Ed. New York, McGraw-Hill, 1974.

[7] WANG, C.Y., BECKERMANN, C., "Prediction of columnar to equiaxed transition during diffusioncontrolled dendritic alloy solidification", Metallurgical and Materials Transactions A, v. 25, n. 5, pp. 10811093, May. 1994.

[8] SPINELLI, J.E., FERREIRA, I.L., GARCIA, A., "Evaluation of heat transfer coefficients during upward and downward transient directional solidification of Al-Si alloys", Structural and Multidisciplinary Optimization, v. 31, n. 3, pp. 241-248, Mar, 2006 
[9] SILVA, J.N., MOUTINHO, D.J., MOREIRA, A.L., et al. "Determination of heat transfer coefficients at metal-mold interface during horizontal unsteady-state directional solidification of $\mathrm{Sn}-\mathrm{Pb}$ alloys", Materials Chemistry and Physics, v. 130, n. 1-2, pp. 179-185, Oct. 2011.

[10] CHEN, L., WANG, Y., PENG, L., et al. "Study on the interfacial heat transfer coefficient between AZ91D magnesium alloy and silica sand", Experimental Thermal and Fluid Science, v. 54, pp. 196-203, Jan. 2014.

[11] ZHANG, L., REILLY, C., LI, L., COCKCROFT, S., et al. "Development of an inverse heat conduction model and its application to determination of heat transfer coefficient during casting solidification", Heat Mass Transfer, v. 50, n. 7, pp. 945-955, Jan. 2014.

[12] SILVA, B.L., CHEUNG, N., GARCIA, A., et al. "Sn-0.7 wt $\% \mathrm{Cu}-(\mathrm{xNi})$ alloys: microstructuremechanical properties correlations with solder/substrate interfacial heat transfer coefficient", Journal of Alloys and Compounds, v. 632, n. 25, pp. 274-285, May. 2015.

[13] NOGUEIRA, M.R., CARVALHO, D.B., MOREIRA, A.L., et al. "Espaçamentos dendríticos primários da liga $\mathrm{Sn}-5 \% \mathrm{~Pb}$ solidificada direcionalmente em um sistema horizontal", Revista Matéria, v. 17, n. 2, pp. 1009 - 1023, May. 2012.

[14] BARROS, A.S., SILVA, A. P., FERREIRA, I. L., et al. "Effect of interfacial heat transfer coefficient on dendritic growth and microhardness during horizontal directional solidification of an aluminum-copper alloy", Defect and Diffusion Forum, v. 367, pp. 10-17, Apr. 2016.

[15] OSÓRIO, W.R., PEIXOTO, L.C., GARCIA, A., "Efeitos da agitação mecânica e de adição de refinador de grão na microestrutura e propriedade mecânica de fundidos da liga Al-Sn”, Revista Matéria, v.14, n. 3, pp. 906-917, Jan. 2009.

[16] CRUZ, K.S., MEZA, E.S., FERNANDES, F.A.P., et al. "Dendritic arm spacing affecting mechanical properties and wear behavior of Al-Sn and Al-Si alloys directionally solidified under unsteady-state conditions”, Metallurgical and Materials Transactions A, v. 41, n. 4, pp. 972-984, Apr. 2010.

[17] SILVA B.L., ARAUJO I., SILVA W.S., et al. "Correlation between dendrite arm spacing and microhardness during unsteady-state directional solidification of Al-Ni alloys", Philosophical Magazine Letters, v. 91, n. 5, pp. 337-343, May. 2011.

[18] DIAS FILHO, J.M., COSTA, T., ROCHA, O.L., et al. "Interconnection of thermal parameters, microstructure and mechanical properties in directionally solidified $\mathrm{Sn}-\mathrm{Sb}$ lead-free solder alloys", Materials Characterization, v. 106, n. 14, pp. 52-61, Aug. 2015a.

[19] DIAS FILHO, J.M., KIKUCHI, R., COSTA, T.A., et al. "Influência das Variáveis Térmicas Sobre os Espaçamentos Dendríticos Terciários durante a Solidificação Direcional Horizontal da Liga Al-6\%Cu", Revista Matéria, v.20, n.1, pp. 47-63, Mar. 2015b.

[20] FAN, J., LI, X., SU, Y., et al. "Dependency of microhardness on solidification processing parameters and microstructure characteristics in the directionally solidified Ti-46Al-0.5W-0.5Si alloy", Journal of Alloys and Compounds, v. 504, n. 1, pp. 60-64, Aug. 2010.

[21] KAYA, H., ÇADIRLI, E., BÖYÜK, U., et al. "Variation of microindentation hardness with solidification and microstructure parameters in the Al based alloys", Applied Surface Science, v. 255, n. 5, pp. 3071 3078, Dec. 2008.

[22] MEZA, E.S., BERTELLI, F., GOULART, P.R., et al. "The effect of the growth rate on microsegregation: experimental investigation in hypoeutectic $\mathrm{Al}-\mathrm{Fe}$ and $\mathrm{Al}-\mathrm{Cu}$ alloys directionally solidified", Journal of Alloys and Compounds, v. 561, pp. 193-200, Jun. 2013.

[23] TEWARI, S.N., GRUGEL, R.N., POIRIER, D.R., "An evaluation of primary dendrite trunk diameters in directionally solidified Al-Si alloys", Metallurgical and Materials Transactions A, v. 45, n. 11, pp. 47584761, Oct. 2014

[24] BRITO, C., REINHART, G., NGUYEN-THI, H., et al. "High cooling rate cells, dendrites, microstructural spacings and microhardness in a directionally solidified Al-Mg-Si alloy", Journal of Alloys and Compounds, v. 636, n. , pp. 145-149, Feb. 2015.

[25] ACER, E., ÇADIRLI, E., EROL, H., et al. "Effect of growth rate on the microstructure and microhardness in a directionally solidified Al-Zn-Mg alloy", Metallurgical and Materials Transactions A, v. 47, n. 6, pp. 3040-3051, Apr. 2016.

[26] GOMES, C.H., KIKUCHI, R.H., BARROS, A.S., et al. "On the natural convection in the columnar to equiaxed transition in directionally solidified aluminum-based binary and multicomponent alloys", Materials Research, v. 18, n. 6, pp. 1362-1371, Dec. 2015. 
[27] SPINELLI, J.E., PERES, M.D, GARCIA, A., "Thermosolutal convective effects on dendritic array spacings in downward transient directional solidification of Al-Si alloys", Journal of Alloys and Compounds, v. 403, n. 1-2, pp. 228-238, Nov. 2005.

[28] ROSA, D., SPINELLI, J., GARCIA, A., "Tertiary dendrite arm spacing during downward transient solidification of Al-Cu and Al-Si alloys", Materials Letters, v. 60, n. 15, pp. 1871-1874, Jul. 2006.

[29] COSTA, T.A., MOREIRA, A.L., MOUTINHO, D.J., et al. "Growth direction and Si alloying affecting directionally solidified structures of Al-Cu-Si alloys", Materials Science and Technology, v. 31, n. 9, pp. 1103-1112, May. 2015.

[30] CARVALHO, D.B., GUIMARÃES, E.C., MOREIRA, A.L., et al. "Characterization of the Al-3wt.\%Si alloy in unsteady-state horizontal directional solidification", Materials Research, v. 16, n. 4, pp. 874-883, May. 2013.

[31] SOUZA, F., LIMA, J., RIZZIOLLI, C., et al. "Microstructure and microhardness in horizontally solidified $\mathrm{Al}-7 \mathrm{Si}-0.15 \mathrm{Fe}-(3 \mathrm{Cu} ; 0.3 \mathrm{Mg})$ alloys", Materials Science and Technology,v. 34, n. 10, pp. 1252-1264, Mar. 2018.

[32] FERREIRA, I.L., MOREIRA, A.L., AVIZ, J.A., et al. "On an expression for the growth of secondary dendrite arm spacing during non-equilibrium solidification of multicomponent alloys: validation against ternary aluminum-based alloys", Journal of Manufacturing Processes, v. 35, 634-650, Oct. 2018.

[33] BARBOSA, C.R., LIMA, J.O., MACHADO, G.M., et al. "Relationship between aluminumrich/intermetallic phases and microhardness of a horizontally solidified AlSiMgFe alloy", Materials Research, v. 22, n. 1, pp. 1-12, Nov. 2018.

[34] FERREIRA, I.L., SPINELLI, J. E., NESTLER, B., et al. "Influences of solute content, melt superheat and growth direction on the transient metal/mold interfacial heat transfer coefficient during solidification of $\mathrm{Sn}-\mathrm{Pb}$ alloys", Materials Chemistry and Physics, v. 111, n. 2-3, pp. 444-454, Oct. 2008.

[35] GÜNDÜZ, M., ÇADIRLI, E., "Directional solidification of aluminium - copper alloys", Materials Science Engineering A, v. 327, n. 2, pp. 167-185, Apr. 2002.

[36] CANTÉ, M.V., CRUZ, K.S., SPINELLI, J.E., et al. "Experimental analysis of the columnar-to-equiaxed transition in directionally solidified $\mathrm{Al}-\mathrm{Ni}$ and $\mathrm{Al}-\mathrm{Sn}$ alloys". Materials Letters, v. 61, n. 11-12, pp. 21352138, May. 2007.

[37] ÇADIRLI, E., "Effect of solidification parameters on mechanical properties of directionally solidified Al-rich Al-Cu alloys", Metals Materials International, v. 19, n. 3, pp. 411-422, May. 2013.

[38] AZEVEDO, H.A., MACHADO, G.M., BARBOSA, C.R., et al. "Microstructural development of an AlNiBi alloy and influence of the transient horizontal solidification parameters on microhardness", Metallurgical and Materials Transactions A, v. 49, n. 10, pp. 4722-4734, Oct. 2018.

\section{ORCID}

José Augusto França Rodrigues Gueber Elias Mendes Santos Júnior André dos Santos Barros Otávio Fernandes Lima da Rocha Antonio Luciano Seabra Moreira https://orcid.org/0000-0001-8102-2719 https://orcid.org/0000-0002-8102-5861 https://orcid.org/0000-0003-1206-9198 https://orcid.org/0000-0002-6037-6662 https://orcid.org/0000-0001-9354-8312 